\title{
Editorial
}

\section{Complement Activation-Related Pseudo-Allergy: A Fresh Look at Hypersensitivity Reactions to Intravenous Iron}

\author{
lain C. Macdougall Katherine Vernon \\ Department of Renal Medicine, King's College Hospital, London, UK
}

Intravenous (IV) iron has been available as a therapeutic agent for well over 50 years, with much of its use preceding the modern-day regulatory control of drugs by agencies such as the FDA, European Medicines Agency and others. In nephrological practice, there was an exponential interest in IV iron coinciding with the introduction of recombinant human erythropoietin in the late 1980 s, when it was realized that, firstly, there was a need for supplemental iron to support increased erythropoiesis, and secondly, oral iron supplementation was largely ineffective in many patients with chronic kidney disease (particularly those on dialysis).

Although unquestionably efficacious in increasing the body's stores of iron, there have always been concerns about the use of IV iron, particularly in relation to allergic or hypersensitivity reactions. The first description of administering parenteral iron to man was in 1932 [1], and the severe and toxic reactions described in this paper led the authors to suggest that extremely low amounts of iron, if any, should be given to humans in this manner. This shortfall was revolutionized with the development of iron preparations in which an iron salt, iron oxyhydroxide, was encased in a carbohydrate shell to allow iron to leach out slowly enough in the circulation to be taken up by circulating transferrin molecules. One of the older IV iron preparations, and the one that started giving IV iron a bad name, was iron dextran. An early report in JAMA [2] reported 3 life-threatening immediate anaphylactic and 8 severe delayed reactions in 471 adult patients and 10 adult prisoner volunteers, who between them received 2,099 administrations of IV iron dextran (Imferon). Imferon was a high molecular weight (HMW) iron dextran preparation, and it is now clear that the incidence of severe immediate hypersensitivity reactions to HMW IV iron compounds (also including Dexferrum) was unacceptably high; both products have subsequently been withdrawn from the market.

The cause of these anaphylactic-type reactions was believed to be IgE-mediated, and classified as a type I hypersensitivity reaction using the classical scheme of Gell and Coombs, originally described in 1968. Patients were known to have circulating anti-dextran antibodies, even before exposure to IV iron, and thus there was a neat biological rationale for this assumption. Other mechanisms were also considered, such as immune complex formation and complement activation, but no evidence was found to implicate these biological pathways [3].

The assumption that immediate hypersensitivity reactions were antibody-mediated persisted with other IV iron preparations, such as iron sucrose and iron sodium

\section{KARGER}

(C) 2016 S. Karger AG, Basel

E-Mail karger@karger.com

www.karger.com/ajn
Prof. Iain C. Macdougall

Consultant Nephrologist and Professor of Clinical Nephrology

Renal Unit, King's College Hospital

London SE5 9RS (UK)

E-Mail iain.macdougall@nhs.net 
gluconate, as well as more modern-day IV irons, ferumoxytol, ferric carboxymaltose and iron isomaltoside 1000. The evidence for this, however, has been at best slender and at worst non-existent. For example, in contrast to the known presence of dextran antibodies in man, there has never been any description of circulating antibodies against sucrose, and this seems biologically implausible.

This has generated a fresh look at what might be causing hypersensitivity reactions to IV iron. A new hypothesis states that some of the reactions may be related to the transient presence of free ('labile') iron in circulation, caused by iron leaching out of the iron-carbohydrate complex too rapidly to be mopped up by transferrin. This was proposed as a possible explanation for why less stable iron-carbohydrate complexes, such as iron sodium gluconate, might cause immediate reactions, particularly if too high a dose was administered too rapidly. Indeed, the administration dose of iron sodium gluconate is lower than for all the other IV iron preparations on the market.

However, more recently, a new concept of what might be causing many (if not the majority) of the hypersensitivity reactions to IV iron has arisen - the concept of complement activation-related pseudo-allergy (CARPA). As the name implies, complement plays a major role in the proposed mechanism of these reactions.

Complement is an ancient part of the innate immune system performing a critical role in the defense against infection, clearance of apoptotic cells and immune complexes, and link with the adaptive immune system. However, it may also mediate tissue injury through the generation of the anaphylatoxins, $\mathrm{C} 3 \mathrm{a}$ and $\mathrm{C} 5 \mathrm{a}$, and the membrane attack complex (C5b-9). It is now recognized that a number of modern-day therapeutic molecules may activate complement via a non IgE-mediated mechanism with the $\mathrm{C} 3 \mathrm{a}$ and $\mathrm{C} 5 \mathrm{a}$ anaphylatoxins binding to mast cells (as well as basophil leucocytes and macrophages), triggering the release of a number of vasoactive mediators that cause the clinical features associated with hypersensitivity reactions. This is the process known as CARPA, and it is now apparent that this is the basis for hypersensitivity reactions to a number of drugs such as monoclonal antibodies (e.g., rituximab), liposome-encapsulated products (e.g., Doxil or AmBisome) and micellarized anti-cancer drugs (e.g., paclitaxel). For example, marked complement activation was seen in cancer patients infused with Doxil, and was correlated with the severity and frequency of hypersensitivity reactions. Furthermore, the rate of drug infusion was critical both in the risk of hypersensitivity reactions and complement activation [4].

CARPA and Hypersensitivity to IV Iron
Is it possible that IV iron infusions could be triggering the same biological process? Given that hypersensitivity reactions to IV iron are much less common than are seen with monoclonal antibodies or liposomal preparations, this is clearly much more difficult to demonstrate. Nevertheless, Hempel et al. [5], in this issue of Am J Nephrol, have attempted to provide some preliminary evidence that CARPA may be implicated in hypersensitivity reactions seen with IV iron, using a number of methods, including complement activation by 5 different IV iron preparations in vitro using functional complement assays, as well as studying complement activation in a group of hemodialysis patients receiving IV ferric carboxymaltose.

In their in vitro assays, iron dextran and ferric carboxymaltose (but not iron sucrose) caused complement activation. In an ex vivo assay, IV iron, particularly iron dextran, significantly increased complement activation in the blood of healthy volunteers and hemodialysis patients. Their data, therefore, suggested that IV iron compounds may have complement-activating potential, and that hypersensitivity reactions to IV iron could potentially be CARPA-mediated.

Clearly, extrapolating these laboratory results into the clinical setting is aspirational, and there was also no consistency regarding the results seen with the various IV iron preparations between the different experimental models. So, the models are not sensitive enough to be able to differentiate between the various IV iron preparations regarding their propensity to develop hypersensitivity reactions.

The design of the experiments by Hempel et al. [5] did not allow the authors to determine whether the complement activation seen was due to the iron itself or the carbohydrate shell. The expanding body of literature on nanoparticles [6], however, suggests that it is more likely to be related to the carbohydrate shell. It would, therefore, be interesting for the authors to repeat their experiments with an isolated carbohydrate shell preparation (i.e., in the absence of iron) to advance this hypothesis.

Furthermore, due to the standard-of-care in their renal unit, the only IV iron preparation tested in their dialysis patients was ferric carboxymaltose. Due to the dialysis procedure itself, there is already a background increase in complement activation. It would have been interesting to test other IV iron preparations in dialysis patients, as well as ferric carboxymaltose administered to a non-dialysis patient population, both using the licensed 6-minute slow bolus injection of $500 \mathrm{mg}$, and perhaps also infusing the same dose over $1 \mathrm{~h}$ to look at comple- 
ment activation in their model. These and other experiments could be considered in the future.

Clearly, given the rarity of hypersensitivity reactions, the ability of IV iron to activate complement is not the only factor that drives hypersensitivity. Other factors that are known to play a part in the development of hypersensitivity include patient characteristics, such as genetics, underlying atopy, old age, rate of drug administration, auto-immune disease, mastocytosis and conditions of pre-existent complement activation.

The study by Hempel et al. [5] has a few limitations, but the authors should be congratulated for providing preliminary data to suggest that CARPA could be implicated in the pathogenesis of IV iron hypersensitivity. It is time for nephrologists to bury the hatchet of IgE-in- duced anaphylaxis seen with the older HMW IV iron dextran preparations, and concentrate on this new mechanism of IV iron-induced hypersensitivity, which we call CARPA. As discussed by Szebeni et al. [7], this has implications for the management of iron reactions, focusing less on drugs such as steroids and anti-histamines, and more on low reactogenic administration protocols.

\section{Disclosure Statement}

Prof. Iain C. Macdougall has received consultancy fees, research support and lecture honoraria from a number of IV iron manufacturers, including AMAG, Vifor Pharma and Pharmacosmos. Dr. Katherine Vernon has nothing to declare.

\section{References}

1 Heath CW, Strauss MB, Castle WB: Quantitative aspects of iron deficiency in hypochromic anemia: (the parenteral administration of iron). J Clin Invest 1932;11:1293-1312.

2 Hamstra RD, Block MH, Schocket AL: Intravenous iron dextran in clinical medicine. JAMA 1980;243:1726-1731.

3 Fleming LW, Stewart WK, Parratt D: Dextran antibodies, complement conversion and circulating immune complexes after intravenous iron dextran therapy in dialysed patients. Nephrol Dial Transplant 1992;7:35-39.
4 Chanan-Khan A, Szebeni J, Savay S, Liebes L, Rafique NM, Alving CR, Muggia FM: Complement activation following first exposure to pegylated liposomal doxorubicin (Doxil): possible role in hypersensitivity reactions. Ann Oncol 2003;14:1430-1437.

5 Hempel CJ, Poppelaarsa F, Gaya da Costa M, Franssen CF, de Vlaam TP, Daha MR, Berger SP, Seelen MA, Gaillard CA: Distinct in vitro complement activation by various intravenous iron preparations. Am J Nephrol 2016; 45:49-59.
6 Szebeni J: Complement activation-related pseudoallergy: a stress reaction in blood triggered by nanomedicines and biologicals. Mol Immunol 2014;61:163-173.

7 Szebeni J, Fishbane S, Hedenus M, Howaldt S, Locatelli F, Patni S, Rampton D, Weiss G, Folkersen J: Hypersensitivity to intravenous iron: classification, terminology, mechanisms and management. Br J Pharmacol 2015;172: 5025-5036. 